\title{
Dobras cutâneas e bioimpedância elétrica perna-perna na avaliação da composição corporal de crianças
}

\author{
Skinfold thicknesses and leg-to-leg bioimpedance \\ for the assessment of body composition in children
}

Monize COCETTI

Sílvia Diez CASTILHO'

Antonio de Azevedo BARROS FILHO²

RE S U M O

\section{Objetivo}

Comparar os componentes da composição corporal, obtidos pela bioimpedância elétrica perna-perna e pela espessura das dobras cutâneas, em crianças.

\section{Métodos}

A composição corporal de 1286 escolares (703 meninas e 538 meninos) entre 7 e 9 anos foi avaliada pelas dobras triciptal e subescapular e pela bioimpedância elétrica perna-perna (TANITA TBF-300). Para analisar a concordância entre o percentual de gordura corporal, a massa gorda e a massa magra obtidos pelos métodos, foram utilizados o coeficiente de correlação de Pearson ( $r$ ) e o modelo de Bland-Altman.

\section{Resultados}

A correlação ( $r$ ) foi alta e significante, respectivamente nas meninas e nos meninos, para o percentual de gordura corporal $(0,77$ e 0,89), para a massa gorda $(0,93$ e 0,94) e para a massa magra $(0,92$ e 0,89). 0 modelo de Bland-Altman também mostrou haver boa concordância entre os métodos, uma vez que poucos pontos, correspondentes às diferenças entre as medidas feitas pelos métodos, estavam fora do intervalo de confiança.

\section{Conclusão}

Estes resultados demonstram que a técnica de bioimpedância elétrica perna-perna é comparável à técnica das dobras cutâneas para avaliar a composição corporal de escolares em estudos populacionais. Individualmente, a ampla variabilidade observada em algumas medidas sugere que a utilização da bioimpedância elétrica perna-perna ou das dobras cutâneas deve ser associada a outros indicadores na avaliação da composição corporal.

Termos de indexação: Composição corporal. Crianças. Impedância elétrica.

\footnotetext{
1 Pontifícia Universidade Católica de Campinas, Centro de Ciências da Vida, Faculdade de Nutrição. Av. John Boyd Dunlop, s/n., Prédio Administrativo, Jd. Ipaussurama, 13059-900, Campinas, SP, Brasil. Correspondência para/Correspondence to: M. COCETTI. E-mail: <mococetti@hotmail.com>

${ }^{2}$ Universidade Estadual de Campinas, Faculdade de Ciências Médicas, Departamento de Pediatria. Campinas, SP, Brasil.
} 


\section{A B S T R A C T}

\section{Objective}

The objective of this study was to compare the components of body composition obtained by leg-to-leg bioimpedance and skinfold thickness measurements in children.

\section{Methods}

Triceps and subscapular skinfold thicknesses and TANITA TBF-300A leg-to-leg bioimpedance were used to determine the body composition of 1286 schoolchildren (703 girls and 583 boys) aged 7 to 9 years. The Pearson's correlation coefficient ( $r$ ) and Bland-Altman plot were used to analyze the agreement between the two methods.

\section{Results}

The two methods correlated strongly and significantly for girls' and boys' percentage of body fat (0.77 and 0.89 , respectively), fat mass (0.93 and 0.94 respectively) and lean mass (0.92 and 0.89 respectively). The BlandAltman plot also showed good agreement between the methods, since only a few points corresponding to the differences between the measurements obtained by the two methods were outside the confidence interval.

\section{Conclusion}

These results show that both methods, triceps and subscapular skinfold thicknesses and leg-to-leg bioimpedance, can be used to asses the body composition of schoolchildren in population-based studies. Yet, the wide variability observed in some measurements suggests that either method should always be associated with other indicators to assess body composition.

Indexing terms: Body composition. Children. Bioelectrical impedance.

\section{N T R O D U ÇÃ O}

O excesso de gordura corporal na infância está associado a doenças cardiovasculares e metabólicas na vida adulta' ${ }^{1}$. No Brasil, assim como em outros países em desenvolvimento, o sobrepeso e a obesidade são as alterações nutricionais mais relevantes nesta faixa etária ${ }^{2,3}$. Desse modo, para auxiliar na identificação e na prevenção primária destes desvios nutricionais, são necessários métodos para avaliar a composição corporal que possam ser empregados com facilidade pelos profissionais de saúde que trabalham com vigilância nutricional.

Técnicas utilizadas para avaliar a composição corporal, como DXA, hidrodensitometria e diluição de isótopos não são apropriadas para estudos populacionais ou para prática clínica, pelo alto custo e pela difícil aplicação, ficando restritas a centros de pesquisa. Nestas situações o uso do método de bioimpedância elétrica ou a medida das dobras cutâneas se tornam ideais pela facilidade de aplicação e pelo baixo custo ${ }^{4,5}$.
Embora não se tenha confirmação direta, presume-se que a gordura subcutânea seja proporcional à gordura corporal total, e que a medida das dobras cutâneas em determinados sítios seja representativa da média da espessura das dobras por toda a superfície do corpo 6 . A medida das dobras cutâneas é um método barato, inócuo ${ }^{7} \mathrm{e}$ rotineiramente empregado na avaliação nutricional de crianças, assim como medidas de peso e altura ${ }^{4}$, porém o método exige treinamento e nem todos estão aptos a realizá-la. Para que os componentes da composição corporal sejam avaliados, estas medidas, uma vez obtidas, devem ser aplicadas a equações, o que demanda tempo e representa uma dificuldade a mais. De outro lado, a análise de bioimpedância elétrica (BIA) utiliza uma corrente elétrica de baixa intensidade que passa pelos tecidos orgânicos e estima a água corporal total e a massa livre de gordura ${ }^{8}$. Eletrodos colocados nas extremidades do corpo, mão-pé bioimpedância horizontal ${ }^{9}$, ou perna-perna - bioimpedância vertical ${ }^{4,5}$ emitem e captam a corrente elétrica. A aplicação do modelo horizontal exige que o avaliado esteja deitado e os eletrodos sejam 
adequadamente posicionados no punho e na mão e no tornozelo e no pé, o que torna o método menos prático e acessível, principalmente para a avaliação de grande número de indivíduos. 0 modelo vertical é portátil e de simples aplicação, pois exige apenas que o indivíduo se coloque em pé, descalço, sobre a plataforma que contém os eletrodos. A BIA perna-perna tem sido utilizada em estudos de avaliação da composição corporal em crianças ${ }^{4,10-12}$.

Embora a medida das dobras cutâneas seja o método normalmente empregado para a avaliação nutricional de crianças, tanto em estudos populacionais como na avaliação nutricional individual, a TANITA perna-perna, pela facilidade de aplicação, pode ser uma alternativa válida para esta população.

Assim sendo, este trabalho tem a finalidade de comparar, em um grupo de crianças brasileiras, os componentes da composição corporal obtidos pela TANITA perna-perna com os obtidos pela espessura das dobras cutâneas.

\section{M É T O D O S}

Este é um estudo transversal que avaliou a composição corporal de 1286 crianças (703 meninas e 583 meninos) entre 7 e 9 anos, matriculadas em escolas públicas e particulares da rede de ensino do município de Campinas (SP), no período de 2002-2003. Participaram da pesquisa os alunos que tiveram o consentimento dos responsáveis e que não apresentavam no momento da avaliação qualquer doença ou limitação que contra-indicasse a tomada das medidas. Todas as crianças foram avaliadas no período da manhã.

O critério de escolha das escolas foi aleatório para as escolas públicas. As particulares foram escolhidas entre as que apresentavam valores de mensalidade semelhantes. No ano de 2003 estavam matriculados 143049 alunos no Ensino Fundamental, em Campinas (SP) ${ }^{13}$. Destes, 120063 freqüentavam escolas públicas e 22986 escolas particulares. Participaram do estudo alunos de 17 entre as 192 escolas públicas e 9 entre as 70 escolas particulares.

De acordo com normas internacionais ${ }^{14}$, foram medidos, por um profissional da saúde previamente treinado, o peso (balança eletrônica TANITA TBF-300A), a altura (antropômetro vertical, modelo Dexter 200), as Dobras Cutâneas Tricipital (DCT) e Subescapular (DCS) (adipômetro Lange), e foi aplicado o método de bioimpedância elétrica perna-perna (TANITA TBF-300A). As crianças usavam roupas mínimas e estavam de pés descalços e em posição ortostática ${ }^{14}$.

As dobras foram medidas três vezes em cada ponto anatômico, no lado esquerdo do corpo, considerando-se como valor representativo da região a média aritmética dessas medidas.

O método da BIA estima a água corporal total e fornece imediatamente os valores do percentual de Gordura Corporal (\%GC), da Massa Gorda (MG) e da Massa Magra (MM).

Para o cálculo do \%GC pela medida da espessura das dobras cutâneas, os valores obtidos foram aplicados às equações de regressão propostas por Slaughter et al. ${ }^{15}$, conforme o valor da soma das dobras fossem, respectivamente < ou >35mm:

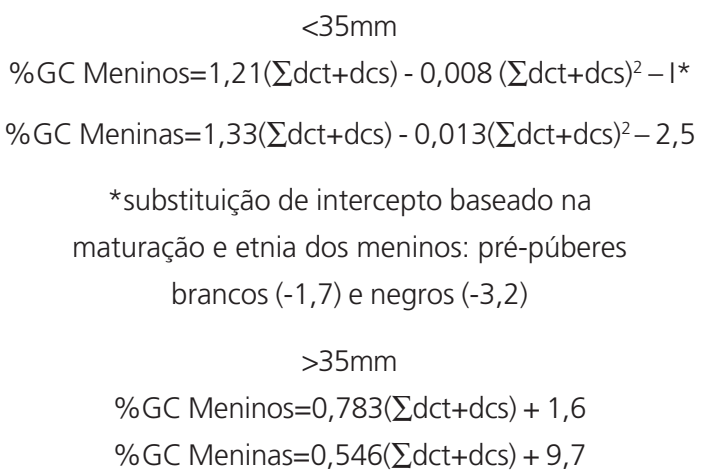

A partir do \%GC foram obtidas a MG e a $M M$, conforme as equações: $M G=\% G C \times$ peso e $\mathrm{MM}=$ peso $-\mathrm{MG}$

Os dados foram armazenados no Software Epi Info versão 6.02 e transferidos para o SAS versão 8.02, no qual foram analisados.

Para verificar a concordância entre os valores do \%GC, MM e MG, obtidos pelo método de 
BIA e das dobras cutâneas foram utilizados o coeficiente de correlação de Pearson ( $r$ ) e o modelo de Bland-Altman ${ }^{16}$. O coeficiente de Pearson expressa a medida da intensidade de associação existente entre duas variáveis quantitativas, e, quanto mais próximo de 1, maior a concordância entre os métodos que estão sendo testados. No modelo de Bland-Altman, a diferença das medidas obtidas pelos dois métodos é alocada ao redor da média. Os dois métodos mostram boa concordância se esta diferença for pequena e 95\% delas caírem dentro de 2 Desvios-Padrão (DP) da média. Quanto mais próxima de zero for a média, menor a chance de existirem vieses. O nível de significância adotado para os testes estatísticos foi de $5 \%(p<0,05)$.

A pesquisa teve a aprovação do Comitê de Ética em Pesquisa da Universidade Estadual de Campinas, a autorização dos diretores das escolas e o consentimento livre e esclarecido dos responsáveis legais dos alunos participantes (protocolo n 277/2001).

\section{RES ULTADOS}

A amostra foi composta de 1286 crianças com idade entre 7 a 9 anos (703 meninas e 583 meninos). A Tabela 1 apresenta média e desvio-padrão para as medidas de peso, altura e Índice de Massa Corporal (IMC), segundo sexo e idade. Todas as médias encontram-se entre os percentis 50 e 85 das novas curvas de referência da Organização Mundial da Saúde ${ }^{17}$.

A Tabela 2 apresenta as associações entre as variáveis percentual de gordura corporal, massa gorda e massa magra, obtidas pelos métodos de BIA perna-perna e dobras cutâneas. A correlação foi alta e significante entre todas as medidas obtidas, principalmente MM e MG para ambos os sexos.

Tabela 1. Caracterização antropométrica de escolares de 7 a 9 anos, segundo o sexo. Campinas (SP), 2002-2003.

\begin{tabular}{|c|c|c|c|c|c|c|c|}
\hline \multirow{2}{*}{$\begin{array}{l}\text { Idade } \\
\text { (anos) }\end{array}$} & \multirow{2}{*}{$n$} & \multicolumn{2}{|c|}{ Peso $(\mathrm{kg})^{*}$} & \multicolumn{2}{|c|}{ Altura $(\mathrm{cm})$} & \multicolumn{2}{|c|}{ IMC $\left(\mathrm{kg} / \mathrm{m}^{2}\right)$} \\
\hline & & $M$ & DP & $M$ & DP & $M$ & DP \\
\hline \multicolumn{8}{|c|}{ Meninas } \\
\hline $7-7,9$ & 202 & 26,2 & 5,7 & 125,0 & 5,7 & 16,7 & 2,6 \\
\hline $8-8,9$ & 227 & 29,8 & 6,5 & 130,7 & 6,2 & 17,3 & 2,9 \\
\hline $9-9,9$ & 274 & 33,2 & 7,4 & 137,0 & 7,0 & 17,5 & 2,8 \\
\hline \multicolumn{8}{|c|}{ Meninos } \\
\hline $7-7,9$ & 157 & 26,9 & 5,9 & 126,2 & 5,8 & 16,7 & 2,7 \\
\hline $8-8,9$ & 189 & 30,7 & 7,5 & 132,2 & 6,3 & 17,5 & 3,2 \\
\hline $9-9,9$ & 237 & 34,8 & 9,1 & 137,4 & 6,5 & 18,2 & 3,6 \\
\hline
\end{tabular}

IMC: índice de massa corporal; M: média; DP: desvio-padrão.

Tabela 2. Medidas descritivas de escolares de 7 a 9 anos, coeficiente de correlação de Pearson ( $r$ ) e significância (p), dos componentes da composição corporal, obtidos por dois métodos (bioimpedância elétrica perna-perna (BIA) e espessura das dobras cutâneas), segundo o sexo. Campinas (SP), 2002-2003.

\begin{tabular}{|c|c|c|c|c|c|c|}
\hline Componente & Método & Sexo $(n)$ & $M$ & DP & $r$ & $p$ \\
\hline \multirow{4}{*}{$\% G C$} & Slaughter et al. ${ }^{15}$ & \multirow{2}{*}{$F(703)$} & 21,34 & 7,22 & \multirow{2}{*}{0,77} & \multirow{2}{*}{$<0,0001$} \\
\hline & $\mathrm{BIA}$ & & 23,85 & 7,59 & & \\
\hline & Slaughter et al. ${ }^{15}$ & \multirow{2}{*}{ M (583) } & 20,34 & 9,67 & \multirow{2}{*}{0,89} & \multirow{2}{*}{$<0,0001$} \\
\hline & BIA & & 18,45 & 8,51 & & \\
\hline \multirow{4}{*}{ MG } & Slaughter et al. ${ }^{15}$ & \multirow{2}{*}{$F(703)$} & 6,84 & 3,96 & \multirow{2}{*}{0,93} & \multirow{2}{*}{$<0,0001$} \\
\hline & $\mathrm{BIA}$ & & 7,60 & 4,12 & & \\
\hline & Slaughter et al. ${ }^{15}$ & \multirow{2}{*}{ M (583) } & 7,10 & 5,65 & \multirow{2}{*}{0,94} & \multirow{2}{*}{$<0,0001$} \\
\hline & $\mathrm{BIA}$ & & 6,47 & 4,91 & & \\
\hline \multirow{4}{*}{ MM } & Slaughter et al. ${ }^{15}$ & \multirow{2}{*}{$F(703)$} & 23,28 & 3,88 & \multirow{2}{*}{0,92} & \multirow{2}{*}{$<0,0001$} \\
\hline & $\mathrm{BIA}$ & & 22,51 & 3,81 & & \\
\hline & Slaughter et al. ${ }^{15}$ & \multirow{2}{*}{ M (583) } & 24,41 & 3,97 & \multirow{2}{*}{0,89} & \multirow{2}{*}{$<0,0001$} \\
\hline & $\mathrm{BIA}$ & & 25,08 & 4,22 & & \\
\hline
\end{tabular}

\%GC: percentual de gordura corporal; MG: massa gorda; MM: massa magra; M: média; DP: desvio-padrão. 

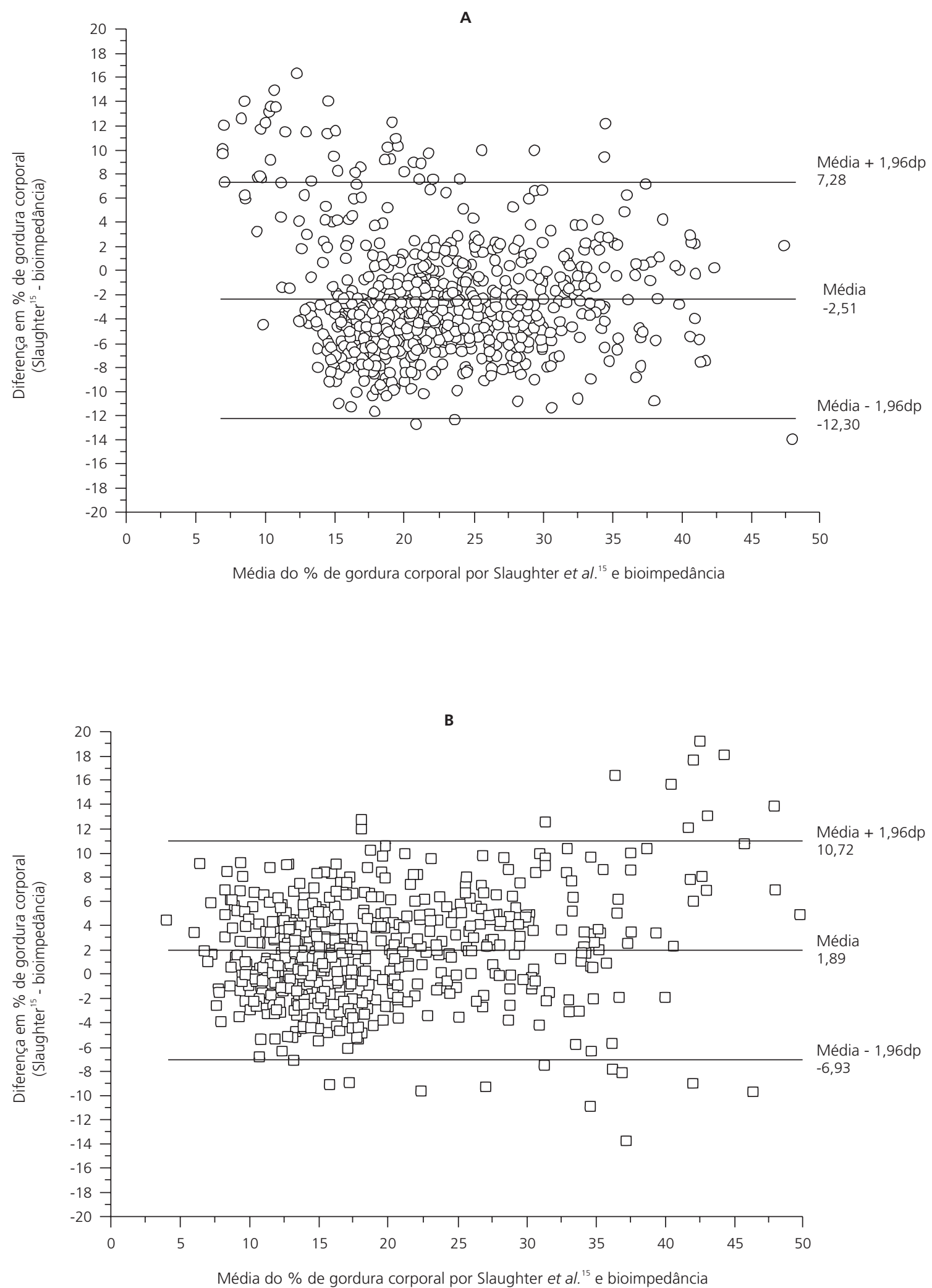

Figura 1. Modelo Bland-Altman ${ }^{16}$ comparando a diferença das medidas do percentual de gordura corporal, obtidas por dois métodos (bioimpedância elétrica perna-perna e espessura das dobras cutâneas), de escolares de 7 a 9 anos dos sexos feminino (A) e masculino (B). Campinas (SP), 2002-2003. 
532 | M. COCETTl et al.
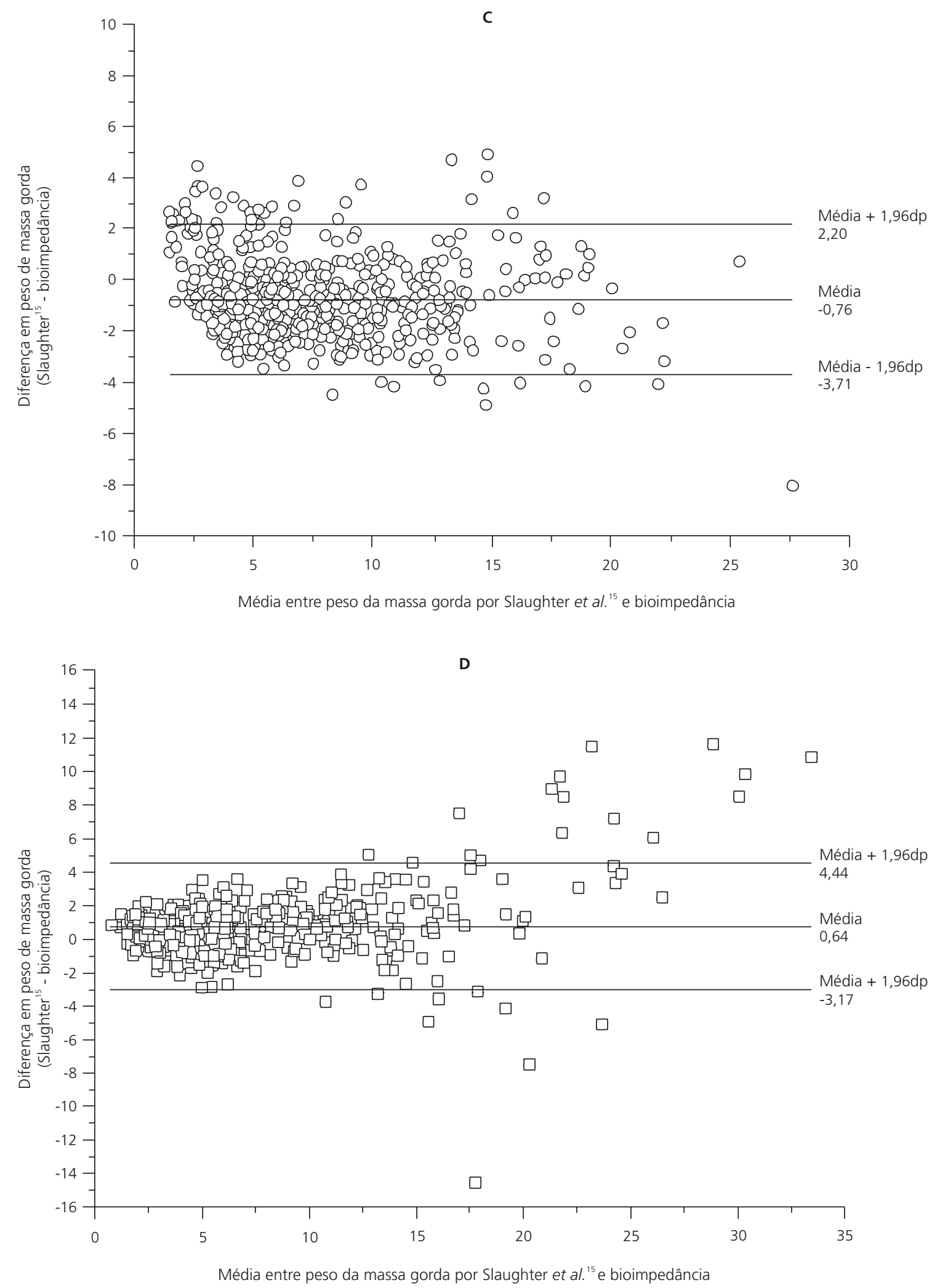

Figura 2. Modelo Bland-Altman ${ }^{16}$ comparando a diferença das medidas da massa gorda obtida por dois métodos (bioimpedância elétrica perna-perna e espessura das dobras cutâneas), de escolares de 7 a 9 anos dos sexos feminino (C) e masculino (D). Campinas (SP), 2002-2003. 

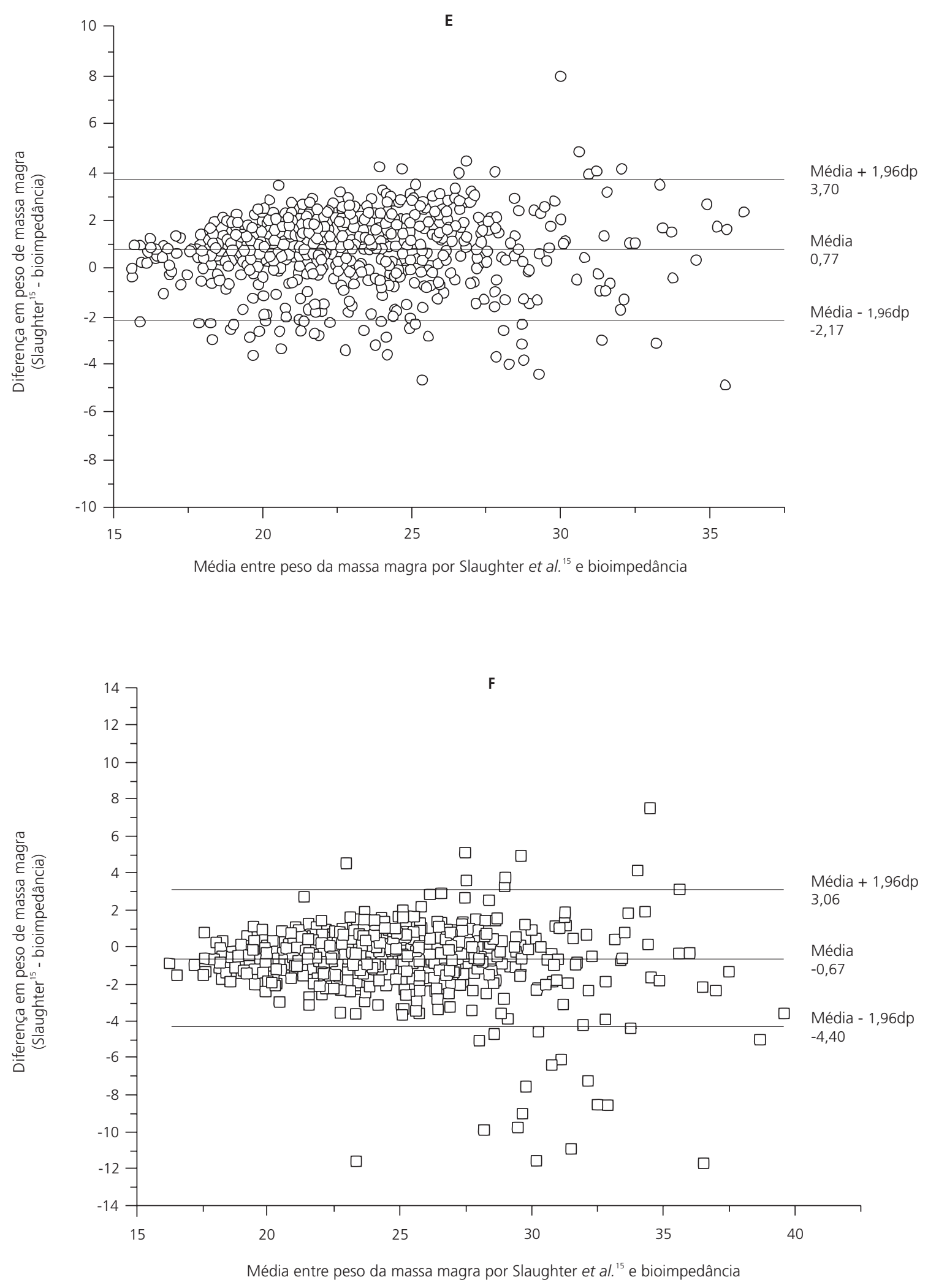

Figura 3. Modelo Bland-Altman ${ }^{16}$ comparando a diferença das medidas da massa magra obtida por dois métodos (bioimpedância elétrica perna-perna e espessura das dobras cutâneas), de escolares de 7 a 9 anos dos sexos feminino (E) e masculino (F). Campinas (SP), 2002-2003. 
534 | M. COCETTI et al.

Nas Figuras de 1 a 3 observa-se o modelo de Bland-Altman ${ }^{16}$, para \%GC, MG e MM, nas meninas e nos meninos, mostrando a diferença nas medidas obtidas pelo método de dobras cutâneas e BIA perna-perna. A média das diferenças entre os métodos foi próxima de zero, principalmente para a massa gorda e a massa magra, em ambos os sexos. Apenas $5 \%$ das diferenças nas medidas dos componentes da composição corporal ficaram fora do intervalo de confiança, para ambos os sexos.

\section{I S C U S S Ã O}

A correlação do percentual de gordura corporal, da massa gorda e da massa magra, obtidos pela medida das dobras cutâneas ou pela BIA perna-perna, foi alta e significante, em ambos os sexos, principalmente para MG e MM. O coeficiente de correlação encontrado neste estudo, para o \%GC obtido pela BIA e pelas dobras cutâneas foi semelhante ao encontrado, por outros autores, em estudos com crianças e adolescentes $^{18,19}$.

A média das diferenças das medidas avaliadas pelo modelo de Bland-Altman foi próxima de zero, respectivamente nas meninas e nos meninos, principalmente para MG e MM, sugerindo que ambos os métodos podem ser usados em estudos populacionais, pois resultam em valores de medida semelhante.

A média das diferenças entre os valores das variáveis obtidas pela subtração Slaughter-BIA, foi negativa para a MG e positiva para a MM nas meninas, parecendo indicar que nelas a BIA tende a superestimar a MG e subestimar a MM. A média positiva para MG e negativa para MM aponta para uma tendência contrária nos meninos; neles a BIA parece subestimar a MG e superestimar a MM. Em estudo brasileiro, com adolescentes do sexo masculino, para validação da BIA perna-perna, os autores observaram que a TANITA tende a superestimar a água corporal total e a massa magra e subestimar a massa gorda em indivíduos com menos água corporal tota ${ }^{20}$. Neste estudo foi observada a associação entre os componentes de composição corporal avaliados pela BIA pernaperna e as dobras cutâneas e os autores concluíram que ambos os métodos podem ser usados em estudos populacionais.

No presente estudo, no entanto, individualmente a variabilidade das diferenças para o \% GC foi muito ampla. Segundo Hammond et al. ${ }^{19}$, esta variabilidade é esperada, pois a impedância avalia o conteúdo de água na massa livre de gordura, enquanto a dobra cutânea é uma medida de gordura corporal, e as duas não se relacionam, necessariamente, de forma linear.

Sung et al. ${ }^{5}$, em estudo de validação da BIA perna-perna com DXA, realizado com crianças chinesas entre 7 e 16 anos, concluíram que a BIA perna-perna é um método alternativo válido, com amplo potencial de uso pela simplicidade, em estudos transversais e longitudinais. Entretanto, estes autores ressaltam que o método superestima o \% GC, o que compromete sua utilização para a avaliação individual, e recomendam que mais estudos sejam realizados para determinar a variação no \% GC obtido por aparelhos de diferentes marcas em diferentes grupos étnicos.

No presente estudo observou-se a mesma tendência, pois a concordância encontrada entre as medidas permite assegurar que para estudos populacionais, a composição corporal de crianças pode ser avaliada, indistintamente, pelos dois métodos. No entanto, os pontos que ficaram fora do intervalo de confiança indicam um fato clinicamente importante em termos individuais. A mesma criança pode ser considerada obesa se avaliada por um método, e com peso adequado se avaliada pelo outro, levando a um diagnóstico nutricional errôneo. Isso aponta para vieses que não puderam ser controlados, uma vez que algumas medidas foram feitas após a ingestão de líquido, enquanto outras após exercício físico. Como a bioimpedância é determinada pela passagem de uma corrente elétrica pelos fluidos do corpo, é fácil perceber que mudanças na hidratação e nos eletrólitos podem ser responsáveis pelas diferenças mais amplas encontradas em 
alguns casos. Tyrrell et al. ${ }^{4}$ também referem que quando se comparam os valores dos componentes da composição corporal obtidos pela BIA perna-perna com os obtidos por métodos de referência, como DXA e hidrodensitometria, geralmente a diferença entre as medidas é baixa e aceitável para grandes grupos populacionais, mas individualmente os erros são significantes, especialmente para o percentual de gordura corporal. Paineau et al. ${ }^{12}$, ao compararem a BIA perna-perna e as dobras cutâneas em crianças francesas, usando diluição de deutério como método de referência, também concluíram que estes métodos são aplicáveis em estudos populacionais, mas apresentam limitações quando empregados para monitoramento individual.

A facilidade de aplicação da bioimpedância elétrica perna-perna torna este método preferível na avaliação da composição corporal de crianças em estudos com grande número de sujeitos, pois requer menos tempo para ser aplicado, se comparado às dobras cutâneas, elimina os erros inter e intra-examinador e não necessita de técnicos treinados para sua execução 7 . Porém, quando a BIA for utilizada para a avaliação da composição corporal é indispensável evitar ao máximo as condições que podem determinar vieses.

Conclui-se que a bioimpedância elétrica perna-perna pode ser utilizada em estudos populacionais para a avaliação da composição corporal de crianças brasileiras desta faixa etária, uma vez que houve alta correlação com as medidas das dobras cutâneas e as médias foram próximas de zero. Entretanto, individualmente, a aplicação não é recomendada, pois as amplas diferenças observadas nas estimativas podem induzir a erros clinicamente importantes. Neste caso, é necessária a aplicação de outros indicadores do estado nutricional associados à avaliação da composição corporal.

\section{A GRADECIMENTO}

À Fundação de Amparo à Pesquisa do Estado de São Paulo, Processo n 2001/04401-2.

\section{COLABORADORES}

M. COCETTI participou da concepção do trabalho, da pesquisa e da redação do texto. S.D. CASTILHO pariticipou da pesquisa e da redação final do artigo. A.A. BARROS FILHO participou da concepção e da redação final do artigo.

\section{REFERÊ NCIAS}

1. Williams SM. Weight and Height growth rate and the timing of adiposity rebound. Obes Res. 2005; 13(6):1123-30.

2. Blossner $M$, Onis $M$. Prevalence and trends of overweight among preschool children in developing countries. Am J Clin Nutr. 2000; 72(4):1032-9.

3. Wang Y, Monteiro C, Popkin BM. Trends of obesity and underweight in older children and adolescents in the United States, Brazil, China, and Russia. Am J Clin Nutr. 2002; 75(6):971-7.

4. Tyrrell VJ, Richards G, Hofman P, Gillies GF, Robinson E, Cutfield WS. Foot-to-foot bioelectrical impedance analysis: a valuable tool for the measurement of body composition in children. Int J Obes Relat Metab Disord. 2001; 25(2):273-8.

5. Sung RYT, Lau P, Yu CW, Lam PKW, Nelson EAS. Measurement of body fat using leg to leg bioimpedance. Arch Dis Child. 2001; 85(3): 263-7.

6. Coward WA, Parkinson SA, Murgatroyd PR. Body composition measurements for nutrition research. Nutr Res Rev. 1988, 1(1):115-24.

7. Heyward VH, Stolarczyk LM. Applied body composition assessment. Champaign (IL): Human Kinetics; 1996.

8. Forbes $\mathrm{G}$. Body composition: influence of nutrition, physical activity, growth and aging. In: Forbes G. Modern nutrition in health and disease. Baltimore: Williams \& Wilkins; 1999.

9. Houtkooper LF, Going SB, Lohman TG, Roche AF, van Loan M. Bioelectrical impedance estimation of fat-free body mass in children and youth: a crossvalidation study. J Appl Physiol. 1992; 72(1): 366-73.

10. Jebb SA, Cole TJ, Dornan D, Murgatroyd PR, Prentice AM. Evaluation of the novel tanita body fat analyser to measure body composition by comparison with a four-compartment model. Br J Nutr. 2000; 83(2): 115-22.

11. Lazzer S, Boirie Y, Meyer M, Vermorel M. Evaluation of two foot-to-foot bioelectrical impedance 
analysers to assess body composition in overweight and obese adolescents. Br J Nutr. 2003; 90(5):987-92.

12. Paineau D, Chiheb S, Banu I, Valensi P, Fontan JE, Gaudelus J, et al. Comparison of field methods to estimate fat mass in children. Ann Hum Biol. 2008; 35(2):185-97.

13. Brasil. Ministério da Educação. Censo educacional. Brasília: MEC; 2003.

14. Lohman TG, Roche AF, Martorell R, editors. Anthropometric standardization reference manual. Champaign (II): Human Kinetics; 1988.

15. Slaughter MH, Lohman TG, Boileau RA, Horswill CA, Stillman RJ, van Loan MD, et al. Skinfold equations for estimation of body fatness in children and youth. Hum Biol. 1988; 60(5):709-23.

16. Bland JM, Altman DG. Statistical methods for agreement between two methods of clinical measurement. Lancet. 1986; 1(8476):307-10.

17. De Onis M, Onyango AW, Borghi E, Siyam A, Nishida C, Siekmann J. Development of a WHO growth reference for school-aged children and adolescents. Bull World Health Organ. 2007; 85(9): 660-7.

18. Goss F, Robertson R, Williams A, Sward K, Abt K, Ladewig $M$, et al. A comparison of skinfolds and leg-to-leg bioelectrical impedance for the assessment of body composition in children. Dyn Med. 2003; 2(1):5.

19. Hammond J, Rona RJ, Chinn S. Estimation in community surveys of total body fat of children using bioelectrical impedance or skinfold thickness measurements. Eur J Clin Nutr. 1994; 48(3): 164-71.

20. Wells JCK, Gigante D, Wright A, Hallal PC, Victora CG. Validation of leg-to-leg impedance for body composition assessment in male Brazilians aged 16-19 years. Int J Body Comp Res. 2003; 1(2):1-6.

Recebido em: 7/11/2007

Versão final reapresentada em: 17/7/2008 Aprovado em: 17/9/2008 